\title{
Les sociétés industrielles étalonnent, mais pourquoi faire?
}

\author{
Par Romain Didelot, Société Delta $\mathrm{Mu}$
}

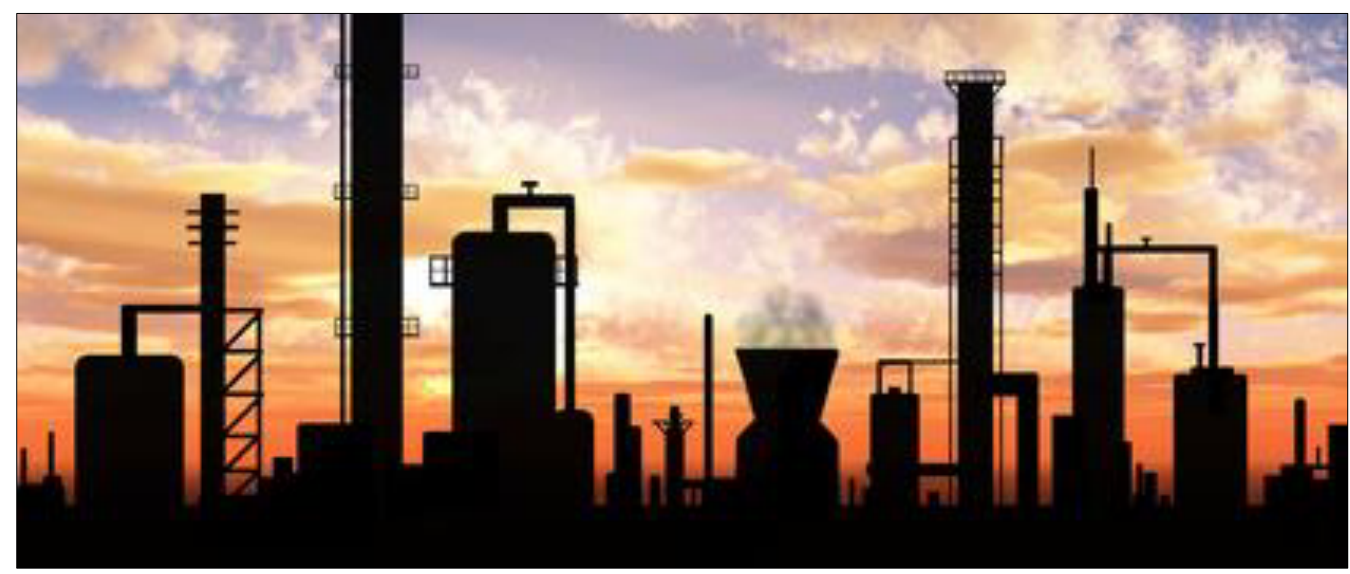

Abstract. The calibration certificate is often not very operational and frequently used only for audits. Can it be different? Can a calibration certificate be used by the manufacturer to improve the quality of measurement and production? The FD X 07-012 provides an exhaustive list of the elements which need to be in the certificate: all the factors that impact the uncertainty of the measurement result have to appear. The central element in this document is the measurement result. The industrials have to verify, on the certificate, the connection chain with the standards measurement, used for the calibration. What counts for the industrialist is the conformity of the measuring instrument. To do so, they need to inspect the measurement results. Nevertheless, the interpretation of the last calibration certificate and the conformity of this operation is not enough. The other results have information which is a shame to miss. In fact, the importance of the measurement standards is to be stable in time. The study of the last calibration result can be weighted in function of the other calibrations results. Finally, the calibration is limited. It cannot certify the quality of the measurement process. In fact, the uncertainty of the measurement result is linked to all the factors influencing the measurement process. The periodical surveillances directly on the measurement process is efficient and enables verification. It can also be an indicator to your calibration intervals.

\section{Introduction}

Dans nombre d'industries, l'unique document faisant foi de la qualité de la mesure est le certificat d'étalonnage, le « saint graal » des auditeurs. Pourtant, en regardant de plus près, le processus de mesure englobe de nombreux facteurs et l'instrument de mesure seul n'en constitue qu'une partie. Alors pourquoi étalonner ses instruments si l'évaluation de la méthode, de l'opérateur, de l'environnement et du mesurande ${ }^{1}$ n'est pas réalisée?
Nous constatons que le certificat d'étalonnage est bien souvent un document pas ou mal exploité. La plupart du temps, il n'est utilisé que pour l'audit. D'un côté, la difficulté à trouver des informations qui intéressent véritablement les industriels constitue une partie de ce défaut. D'un autre côté, il est fréquent que la quantité d'instruments gérés par le métrologue lors de chaque campagne puisse le handicaper dans l'exercice de ses fonctions. Peut-il en être autrement ? Un certificat d'étalonnage peut-il être utilisé par l'industriel pour améliorer la qualité des mesures et donc de sa production?

\footnotetext{
${ }^{1}$ Grandeur que l'on veut mesurer

Email de correspondance de l'auteur : rdidelot@deltamu.fr
} 
Dans une première partie, nous nous intéresserons aux certificats d'étalonnage et à la signification de toutes les informations qui doivent apparaitre dans ces documents. Dans une deuxième partie, nous parlerons de la manière dont ces informations sont utilisables par les industriels. Enfin, nous développerons les informations utiles dans un Certificat d'étalonnage et les opérations complémentaires nécessaires pour garantir au maximum la validité du processus de mesure.

\section{1 - Les informations du Certificat d'étalonnage}

Le Certificat d'étalonnage est encadré par le fascicule FD X 07-012 qui se définit comme suit : "Le présent fascicule de documentation a pour objet de préciser le contexte d'application d'un étalonnage et de définir le contenu du document émis à l'issue de cet étalonnage. ". Mais qu'est-ce qu'un étalonnage ?

Le VIM ${ }^{2}$ le définit ainsi :

\section{« 2.39 (6.11) étalonnage,}

opération qui, dans des conditions spécifiées, établit en une première étape une relation entre les valeurs et les incertitudes de mesure associées qui sont fournies par des étalons et les indications correspondantes avec les incertitudes associées, puis utilise en une seconde étape cette information pour établir une relation permettant d'obtenir un résultat de mesure à partir d'une indication NOTE 1 Un étalonnage peut être exprimé sous la forme d'un énoncé, d'une fonction d'étalonnage, d'un diagramme d'étalonnage, d'une courbe d'étalonnage ou d'une table d'étalonnage. Dans certains cas, il peut consister en une correction additive ou multiplicative de l'indication avec une incertitude de mesure associée.

NOTE 2 Il convient de ne pas confondre l'étalonnage avec l'ajustage d'un système de mesure, souvent appelé improprement "auto-étalonnage», ni avec la vérification de l'étalonnage.»

\section{Le fait d'avoir un instrument étalonné signifie, pour beaucoup d'utilisateurs, avoir un instrument juste et fidèle. Or il n'en est rien.

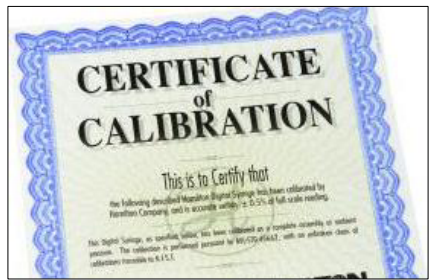 L'étalonnage ne fait que constater des écarts, il n'a pour objet, ni de juger ces écarts, ni Illustration 2 : Certificat of de les corriger même si la calibration \\ nouvelle définition intègre dans l'opération d'étalonnage la modélisation des résultats donc, le cas échéant, la possibilité de corriger. Force est de constater qu'à ce jour, peu d'étalonnages respectent formellement la dernière partie de cette définition.}

Le fascicule de documentation FD X 07-012 est explicite quant aux éléments qui doivent être pris en compte dans le certificat d'étalonnage. Il s'agit entre autres

${ }^{2}$ Vocabulaire International de Métrologie des informations concernant le(s) moyen(s) d'étalonnage, le moyen de mesure étalonné, l'opérateur ayant réalisé la mesure, la procédure utilisée et les conditions environnementales. En effet, tous les facteurs influant sur l'incertitude du résultat d'étalonnage doivent apparaître ainsi que la date d'étalonnage. L'élément central dans ce document est le résultat de l'étalonnage.

Parmi toutes ces informations, la confusion est souvent possible, notamment lorsque les documents sont organisés de façons différentes. La date d'émission avec la date de réalisation de l'étalonnage, l'expression de l'écart et de la correction d'un instrument de mesure, la désignation du moyen sur le certificat avec celle de l'industriel sont autant de facteurs pouvant apporter de la confusion lors de l'exploitation des documents. Tout d'abord dans l'optique du raccordement aux étalons internationaux (ou à un référentiel commun défini avec le client), l'industriel doit vérifier le raccordement documentaire aux étalons nationaux et internationaux sur le document d'étalonnage. Comme il est fréquent que l'objectif principal soit de vérifier la conformité de son moyen, il lui faut inspecter les résultats de mesure. Dans bien des cas, les constats de vérification sont établis sur des critères normatifs ou constructeurs, qui ne correspondent pas nécessairement aux besoins réels du processus de mesure. Il convient bien sûr de porter attention à l'incertitude du résultat d'étalonnage. Celle-ci devra être prise en compte dans un calcul d'incertitude du processus de mesure qui mettra en œuvre le moyen étalonné (sauf si la vérification prend en compte ladite incertitude). Elle permet également de vérifier la capabilité avec laquelle le laboratoire établi la conformité de l'instrument.

Il n'est en effet pas rare de voir des incertitudes plus grandes que les limites de tolérances.

En témoigne l'exemple sur les cales étalons.

Prenons une cale de $500 \mathrm{~mm}$ classe 1, ayant une tolérance $\mathrm{de}+/-$ 2,2 $\mu \mathrm{m}$ sur sa longueur selon l'ISO 3650

Pour ce type de cale, l'analyse de la moyenne des incertitudes annoncées par les laboratoires accrédités COFRAC nous permet d'avancer une incertitude moyenne de $+/-2.0 \mu \mathrm{m}$ à deux incertitudes-types composées. Notons que certains laboratoires annoncent même jusqu'à $4 \mu \mathrm{m}$ d'incertitude ${ }^{3}$.

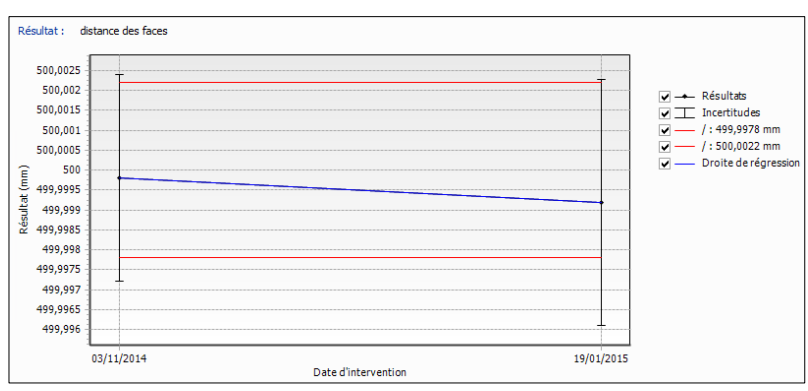

Figure 1: Exemple d'incertitudes d'étalonnage sur une cale étalon de classe 1 par deux laboratoires différents.

Dans cet exemple, les incertitudes de mesure sont plus larges que les tolérances normatives, ce qui ne donne que

\footnotetext{
${ }^{3}$ Moyenne prise sur 12 laboratoires sur le site du COFRAC
} 
peu de valeur à la conformité de cette cale à la classe 1 . Même à l'issus de deux étalonnages réalisés par deux laboratoires différents.

\section{2 - Améliorer la qualité du résultat}

Il est important de ne pas s'arrêter à l'interprétation $\mathrm{du}$ dernier certificat d'étalonnage et à la conformité déclarée sur cette dernière opération. En effet, les précédents étalonnages contiennent de

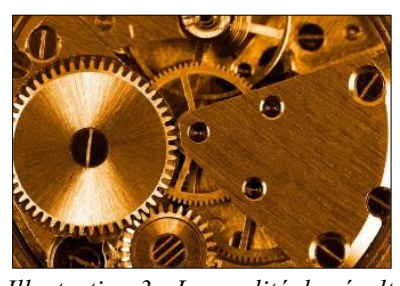
précédents certificats peut permettre d'étudier l'éventuelle dérive de l'étalon et ainsi, d'ajuster la périodicité d'étalonnage, ou même d'anticiper une non-conformité sur le moyen. Ensuite, les opérations d'étalonnage ne sont pas parfaites et présentent une certaine incertitude. L'étude des résultats du dernier étalonnage peut être pondérée en fonction des résultats précédents. Ceci afin d'avoir une information plus juste sur la valeur de l'étalon.

On peut aussi travailler sur une étude plus globale des résultats d'étalonnage afin d'en tirer des informations sur l'évolution des moyens dans l'entreprise. Il existe par exemple des méthodes de détermination des périodicités d'étalonnage dans la FD X 07-014 dans la rubrique intitulée : "Méthode de la dérive ». Ces méthodes consistent à étudier les dérives individuelles des instruments sur un parc d'instruments de mesure par type d'utilisation ou par type de moyen afin d'en estimer une dérive maximale.

Donc pour optimiser les résultats de l'étalonnage, il est

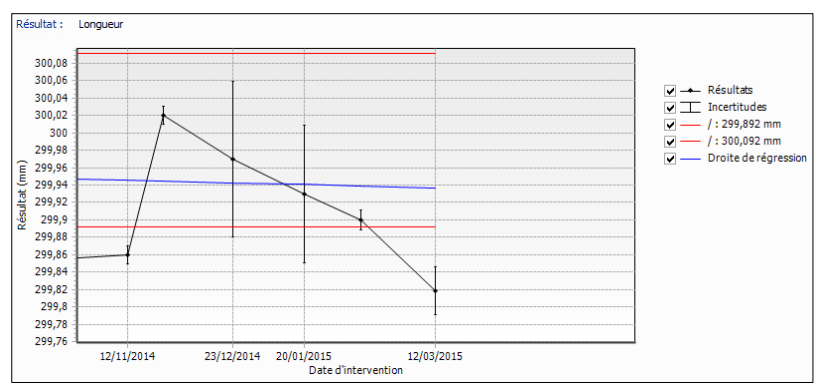

important de renseigner les résultats à l'aide d'un logiciel de gestion ou d'un tableur. Une étude statistique adaptée augmenterait considérablement la valeur des résultats d'étalonnage du moyen de mesure. Cette méthode permet aussi de pouvoir évaluer les compétences d'un laboratoire d'étalonnage, notamment en isolant les résultats aberrants ou « douteux ».

Prenons l'exemple d'un calibre à mâchoire :

Figure 2 : Historique d'étalonnage d'un calibre à mâchoire
L'analyse de la figure 2 nous amène à penser que

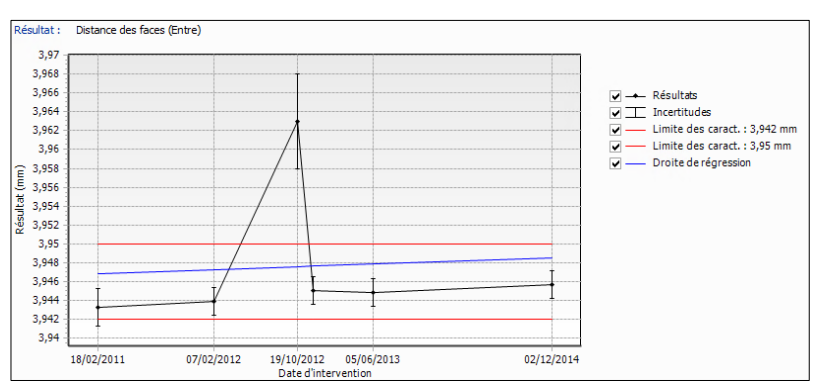

l'étalonnage réalisé le 19/10/2012 est aberrant. En effet, une évolution positive du coefficient directeur de l'usure du calibre à mâchoire est un phénomène normal dans son utilisation. Dans cet exemple, une non-conformité est identifiée sur le calibre à mâchoire, indiquant une usure anormalement élevée. Une contre mesure est réalisée, révélant une erreur dans l'étalonnage précédent. Il est intéressent de savoir que même un étalonnage sous accréditation COFRAC ne garantit pas une mesure fiable. En effet, ISO/CEI 17025 donne les « Exigences générales concernant la compétence des laboratoires d'étalonnage et d'essais ». En effet, un certificat portant le logo type COFRAC reconnait la compétence du laboratoire à réaliser l'essai mais ne peut pas garantir tous les résultats de mesures réalisés par le laboratoire (GEN REF 11, p 6.1).

\section{3 - Compléter l'étalonnage : une nécessité}

L'étalonnage a ses limites. Il ne peut à lui seul assurer la qualité du processus de mesure. En effet l'incertitude sur le résultat de mesure du processus est liée à tous les facteurs influant sur le processus de mesure :

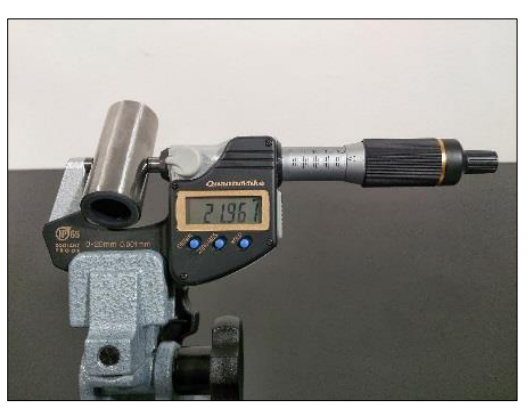

Illustration 4 : Mesure au micromètre d'extérieur
La méthode
L'opérateur
Les conditions ambiantes
Le moyen de mesure
Le mesurande

Il est conseillé dans le Guide du $\mathrm{CFM}^{4}$ " surveillance des processus de mesure » de réaliser des surveillances ou vérifications régulières directement au niveau du processus de mesure. Ces surveillances peuvent être documentées et exploitées de la même manière que les résultats d'étalonnage. Cette action est souvent simple, efficace et permet une vérification directe sur la mesure. La surveillance peut même constituer un indicateur pour la périodicité d'étalonnage.

Exemple de cet indicateur qui permet d'anticiper, via une simple carte de contrôle, une dérive du processus de mesure et donc de prévenir une non-conformité.

\footnotetext{
${ }^{4}$ Collège Français de Métrologie
} 
Figure 3 : Surveillance d'un processus de mesure en dérive

Le guide CFM " surveillance des processus de mesure " indique plusieurs méthodes :

1) Le suivi d'un objet connu

2) La redondance des mesures

3) Le processus de mesure dédié

4) La corrélation/Caractère intrinsèque des caractéristiques « objet »

5) Le cas de l'utilisation d'objet Conforme et Non Conforme

Il est conseillé de faire réaliser ces surveillances par les opérateurs de production. Ce qui permet de développer les compétences de ces derniers. De plus, les opérateurs qui utilisent ces moyens tous les jours, s'intéressent souvent aux performances et à l'utilité de leurs actions. L'opacité des résultats de l'étalonnage, bien gardés et documentés au service métrologie, ne sert pas toujours la qualité de la mesure. Parfois même, l'étalonnage peut conduire à l'effet inverse : «Puisque mon instrument est conforme jusqu'à telle date, je n'ai plus besoin d'y porter attention ».

Concernant la détermination des périodicités d'étalonnage, la norme : «ILAC-G24 / OIML D 10 » va même plus loin sur les Méthode de vérification « en service " ou test de " boîte noire ». Cette méthode est conseillée, dans la norme, pour les instruments complexes ou les consoles de test. Elle consiste à réaliser spécialement une « boîte noire » qui permettra de vérifier les paramètres choisis de notre équipement. La vérification peut être faite en direct sur l'équipement sans réaliser d'étalonnage. Si l'équipement est mesuré par la «boîte noire » en dehors de l'EMT $^{5}$, il est renvoyé en étalonnage pour un étalonnage complet.

$\mathrm{Au}$ final, la périodicité d'étalonnage n'existe plus ici, l'étalonnage n'est déclenché qu'en cas de soucis détecté suite à une surveillance.

\section{Conclusion}

Il est assez normal que l'étalonnage à lui seul ne soit pas gage de la qualité des mesures pour une société industrielle. Il est un maillon d'une chaine de mesure qui participe à prendre des décisions sur la qualité du produit fini. Les autres facteurs sont souvent malheureusement oubliés car difficiles à évaluer. Et pourtant, ils ne sont pas négligeables dans la plupart des cas. Un étalonnage intelligent et raisonné de son parc d'instruments de mesure peut être, s'il est bien exploité, un facteur participant de manière significative à l'amélioration de la qualité dans l'industrie.

Les différents facteurs pour atteindre l'efficience industrielle passe par l'étalonnage, mais aussi, l'étude des tolérances, l'optimisation des périodicités d'étalonnage, le calcul de l'incertitude de mesure et la mise en place de surveillances et de vérifications sur le processus de mesure. Ces facteurs permettront, tous ensemble,

${ }^{5}$ Ecart Maximal Toléré d'augmenter la performance du processus de mesure et donc de réduire les coûts tout en augmentant la qualité du produit.

En France nous comptons environ 230000 sociétés industrielles 6 . Se posent-elles ces questions ou appliquentelles des dispositions considérées réglementaires? Quoi qu'il en soit, le facteur temps est une donnée qui est très importante dans l'industrie. La rentabilité d'un processus de fabrication comprend la main d'œuvre et les acteurs intervenants autour du produit ou du service. Tout ce qui peut faire gagner du temps est une variable importante à prendre en compte. C'est le cas par exemple de la réduction des étalonnages " inutiles » ou des non conformités aberrantes par l'établissement d'une vraie optimisation des périodicités d'étalonnage de son parc. De plus, la recherche de critères de tolérances adaptés peut aider grandement la rentabilité d'un service métrologie ou qualité. Et pourquoi ne pas s'intéresser ensuite aux facteurs d'incertitudes importants dans un processus de fabrication? 